\title{
Accurate wave functions in the presence of long-ranged potentials
}

\author{
George Rawitscher \\ ${ }^{1}$ Physics Department, University of Connecticut, Storrs CT 06269, USA
}

\begin{abstract}
For attractive potentials of the form $1 / r^{3}$ or $1 / r$ obtaining accurate wave functions at long distances can be numerically challenging because of the many oscillations involved. In this case high accuracy can be achieved using the phase-amplitude representation of the one-dimensional wave function.
\end{abstract}

For the cases where the attractive potentials are of the $1 / r^{3}$ or $1 / r$ forms (dipole dipole interaction between atomic molecules, or, the Coulomb potential, respectively), and also for the solution of threebody clusters with halo conditions, accurate wave functions at large distances are required. The usual procedure is to solve the Schrödinger equation, and follow the wave function $\psi(r)$, with all its many oscillations, from small distances to large ones, accumulating large errors in the process.

A way to bypass this difficulty is to resort to the phase-amplitude ( $\mathrm{Ph}-\mathrm{A})$ representation of the one-dimensional wave function. This representation has the form $\psi(r)=y(r) \sin [\phi(r)]$, where $y$ and $\phi$ are the amplitude and phase, respectively. The main advantage is that both quantities are nearly monotonic functions of the distance $r$, hence their calculation requires far fewer mesh points than the wave function itself. One difficulty is that the differential equation for $y$, given by W. E. Milne in 1930 [1], has a non-linear term of the form $1 / y^{3}$. That equation was subsequently solved iteratively by Seaton and Peach in 1963 [2] in the form

$$
\frac{k}{y_{n+1}^{2}}=\left[w+D_{n}\right]^{1 / 2}, \quad n=0,1,2, \ldots
$$

where $D(r)=\left(d^{2} y / d r^{2}\right) / y$, and $w(r)=k^{2}-V$. Here $V$ is the central potential assumed to be attractive, and $k$ is the wave number. The factor $\hbar^{2} / 2 m$ has already been taken into account for the potential and the energy $E=k^{2}$, so that both are now given in units of inverse length squared. Once $y$ is known, the phase can be obtained according to $\phi(r)=k \int_{0}^{r}\left[y\left(r^{\prime}\right)\right]^{-2} d r^{\prime}$. For $n=0$ one sets $D_{0}=0$, and $y_{1}$ is the WKB approximation.

The present approach consists in expanding the amplitude $y$ in terms of Chebyshev polynomials, $y(x)=\sum_{s=0}^{N} a_{s} T_{s}(x), \quad-1 \leq x \leq 1$. In order to avoid having to calculate the derivatives of $y$, the quantities $D_{n}$ are obtained recursively by means of

$$
D_{n+1}=\frac{5}{16} \frac{\left(V^{\prime}\right)^{2}}{\left(w+D_{n}\right)^{2}}+\frac{1}{4} \frac{V^{\prime \prime}}{\left(w+D_{n}\right)}, \quad n=0,1,2, \ldots
$$

This equation can be solved non-iteratively in terms of the roots of a cubic equation [3] which is very fast (by a factor 10-20) compared to the conventional methods of solving the Schrödinger 


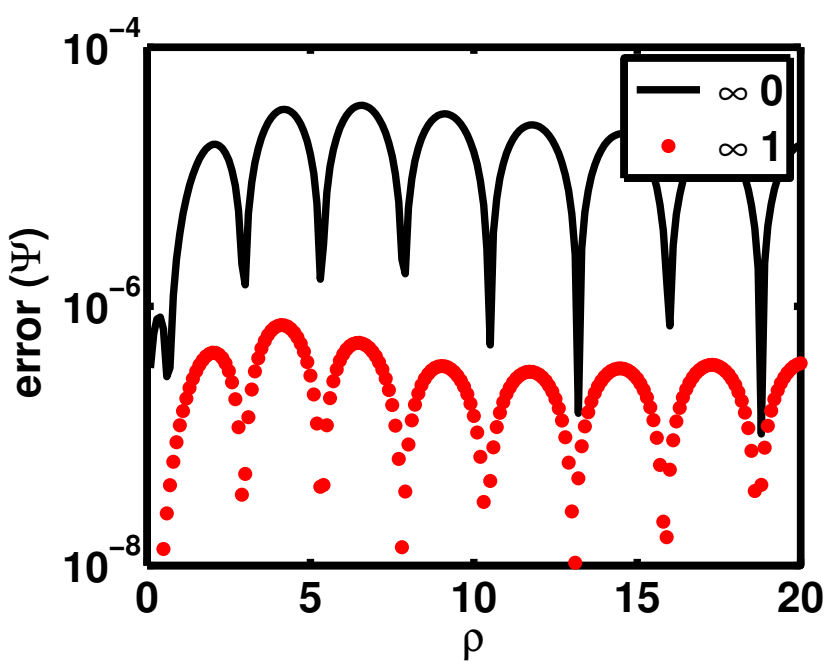

Figure 1. The accuracy of the wave function for a Coulomb potential with $\eta=-2$, calculated in the radial interval $[0,200]$. The result labeled $\infty 0$ is based on Eq. (2), while for $\infty 1$ the first order derivative of $D_{n}$ is included in that equation.

equation. If the derivatives of $D$ are included in Eq. (2), then the accuracy of the Ph-A result is increased considerably, as is illustrated in Fig. 1 for the case of a Coulomb potential "rounded" [3] near $r=0$, with $\eta=-2$ and $N=100$, calculated in the radial $\rho=k r$ interval 0 to 200 .

In summary, the computational time for the $\mathrm{Ph}-\mathrm{A}$ wave function is much faster than for conventional methods, the number of support points is also much smaller, the asymptotic value of the amplitude is automatically equal to unity, and the $\mathrm{Ph}-\mathrm{A}$ calculation can be carried out for long range potentials to large distances. A generalization to repulsive potentials is underway.

\section{References}

[1] W. E. Milne, Phys. Rev. 35 (1930) 863;

[2] M. J. Seaton and G. Peach Proc. Phys. Soc. 79 (1962) 1296 doi:10.1088/0370-1328/79/6/127;

[3] G. Rawitscher, A Spectral Phase-Amplitude method for propagating a wave function to large distances, Comp. Phys. Comm. 191 (2015) 33 